\title{
Determination of the Degree of Cross-linking and Curing with Single-sided NMR
}

\author{
Norbert Halmen, Christoph Kugler, Thomas Hochrein, Peter Heidemeyer, Martin Bastian \\ SKZ - German Plastics Center, Friedrich-Bergius-Ring 22, 97076 Wuerzburg, Germany \\ n.halmen@skz.de
}

\begin{abstract}
:
The degree of cross-linking and curing is one of the most important values concerning the quality of cross-linked polyethylene (PE-X) and the functionality of adhesives. Up to now, the measurement of this property is mostly time-consuming and usually destructive. Within a research project the usability of single-sided NMR for the non-destructive determination of the degree of cross-linking and curing as process monitoring was observed. First results indicated the possibility to distinguish PE-X samples with different degrees of cross-linking. Also the homogeneity of the samples was studied. Furthermore the curing kinetics of adhesives can also be monitored. The measurements show good agreement with reference tests (wet chemical analysis, Differential Scanning Calorimetry, Dielectric Analysis). Also the influence of sample temperature on the characteristic relaxation times can be seen.
\end{abstract}

Key words: NMR, PE-X, cross-linking, curing, non-destructive

\section{Cross-linking and Curing}

Cross-linking of commodity plastics is a common method to achieve mechanical, thermal and chemical properties comparable to those of high-performance polymers [1]. Crosslinked polyethylene (PE-X) and polyamide hold the biggest market share. Moreover, the market segments of thermoset injection molding, fiber reinforced composites and resin-based adhesives are steadily growing. Although processing time in the production of crosslinked polymers is continuously decreasing the degree of cross-linking/curing is still measured in the laboratory [2]. Relatively long measurement times for a quantitative result and the destructive character of these standard test methods inhibit a $100 \%$ process monitoring. The radicals necessary for the cross-linking of PE can be generated either by the use of special additives and energy input $(\mathrm{PE}-\mathrm{Xa} / \mathrm{Xb} / \mathrm{Xd})$ or radiation (PE-Xc). For thermosets the reaction is started by adding the curing agent or the necessary activation energy.

\section{Single-Sided NMR}

An innovative approach to overcome the drawback of destructive testing is provided by the nuclear magnetic resonance (NMR) technique with test systems for a single-sided access. Such devices with inhomogeneous stray fields and specially adapted radiofrequency (rf) resonators can be used for NMR relaxometry [3]. Several magnets are arranged in a way that the magnetic field $B_{0}$ is parallel to the surface at a fixed position above the rf coil. The samples to be tested are placed or moved through this so-called sensitive volume.

\section{Principles of NMR relaxation}

The loss of nuclear magnetization is known as relaxation. The two main reasons are the dissipation of energy from the spin system to the molecular environment and the coherence loss of a high quantity of precessing spins. Within the formalism of the Bloch equations [4], for systems with spin $1 / 2$, these two processes are described by the relaxation times $T_{1}$ for longitudinal (or spin-lattice) relaxation and $T_{2}$ for transverse (or spin-spin) relaxation, respectively. For spin systems of higher order there are further relaxation times. Spin interactions as well as time scale and geometry of molecular motion determine the relaxation times in a certain spin system [5]. Therefore relaxation times and their variations are adequate indicators for the state of a material when compared with a reference.

In the case of polymers mainly the transverse relaxation time $\left(T_{2}\right)$ of the sample is crucial. This parameter is influenced by the molecular motion of the polymer chains and therefore sensitive to the physical environment of the studied molecule. Due to cross-linking the degrees of freedom of molecules in amorphous regions are reduced by the formation of a network and a change in relaxation times 
occurs. The feasibility to correlate NMR relaxation with the degree of cross-linking and curing has already been shown $[6,7,8,9]$.

\section{Relaxation Times and Echo Sums}

In the given measurement geometry the observation of the free induction decay is not possible since the relaxation times of most polymers are shorter than the dead time of the rf coil. To measure the transverse relaxation by single-sided NMR, spin echoes are generated which refocus the magnetization dephasing caused by inhomogeneities of the magnetic fields. For systems with spin $1 / 2$ a single echo can be generated by applying two rf pulses as in the Hahn echo sequence [10]. Instead of applying several single echoes with different echo times to get the transverse magnetization through the attenuation of the echo maxima, a multi-echo sequence is used. The commonly used multi-echo train is the one after Carr, Purcell, Meiboom and Gill (CPMG) [11, 12], which uses short echo times and allows the measurement of multiple echo maxima at different echo times in a single shot. However, since the magnetic field is not perfectly homogeneous, the relaxation time $T_{2}$ is actually an effective relaxation time $T_{2 \text { eff }}$ because of offresonance effects and flip angle variations in the inhomogeneous $B_{0}$ and $B_{1}$ (applied by rf coil) fields [3].

The transverse relaxation of semi-crystalline polymers can be described by two components, a rapidly relaxing one deriving from crystalline and rigid amorphous areas and a slowly relaxing one from mobile amorphous regions [3]. The measurement signals can be fitted by:

$f(t)=A_{\text {short }} e^{-\frac{t}{T_{2, \text { short }}}}+A_{\text {long }} e^{-\frac{t}{T_{2, \text { long }}}}$

Another way to get suitable information is the use of echo sums [13], also known as cumulative binning. The signal can be approximated by a sum of exponential functions

$s(t)=\sum_{i} A_{i} e^{-\frac{t}{T_{2 e f f, i}}}$.

The echo sum is proportional to the signal integral which, after normalization of $s(t)$, represents an amplitude-weighted average of the relaxation time $T_{2 \text { eff }}$ [3]:

$\int_{0}^{\infty} \frac{s(t)}{s(0)} d t=\sum_{i} w_{i} T_{2 e f f, i}=\left\langle T_{2 e f f}\right\rangle$

where $w_{i}$ are the relative amplitudes of the components.

Stable magnet and sample temperature are important for the reproducibility of the measurements. On the one hand the relaxation times $\left(T_{1}\right.$ and $\left.T_{2}\right)$ of plastics are strongly influenced by temperature and on the other hand the position of the sensitive volume varies with the temperature of the magnet.

\section{Measurement Setup}

Here, a commercially available single-sided NMR system (NMR-Mouse PM5 by Magritek $\mathrm{GmbH}$ ) was used to test the potential of this technique for quantifying the degree of crosslinking and curing within short measurement times while delivering depth information. Important specifications are given in Tab. 1. The magnetic system was used in combination with the spectrometer $\mathrm{Kea}^{2}$, also a product from Magritek $\mathrm{GmbH}$. The complete measurement setup is shown in Fig. 1.

Tab. 1: Specifications of the used NMR-Mouse PM5 by Magritek $\mathrm{GmbH}$.

\begin{tabular}{|c|c|}
\hline Field strength & $0.45 \mathrm{~T}$ \\
\hline Operating frequency & $20.17 \mathrm{MHz}$ \\
\hline Gradient strength & $1030 \mathrm{kHz} / \mathrm{mm}$ \\
\hline Rf coil lateral size & $13 \times 13 \mathrm{~mm}^{2}$ \\
\hline $\begin{array}{c}\text { Maximum } \\
\text { measurement depth }\end{array}$ & $5.3 \mathrm{~mm}$ \\
\hline Dead time & $11 \mu \mathrm{s}$ \\
\hline $\begin{array}{c}\text { Spacer between coil } \\
\text { plate and magnet } \\
\text { system }\end{array}$ & thickness $2 \mathrm{~mm}$ \\
\hline $\begin{array}{c}\text { Resolution (depth } \\
\text { direction) }\end{array}$ & $>10 \mu \mathrm{m}$ \\
\hline
\end{tabular}

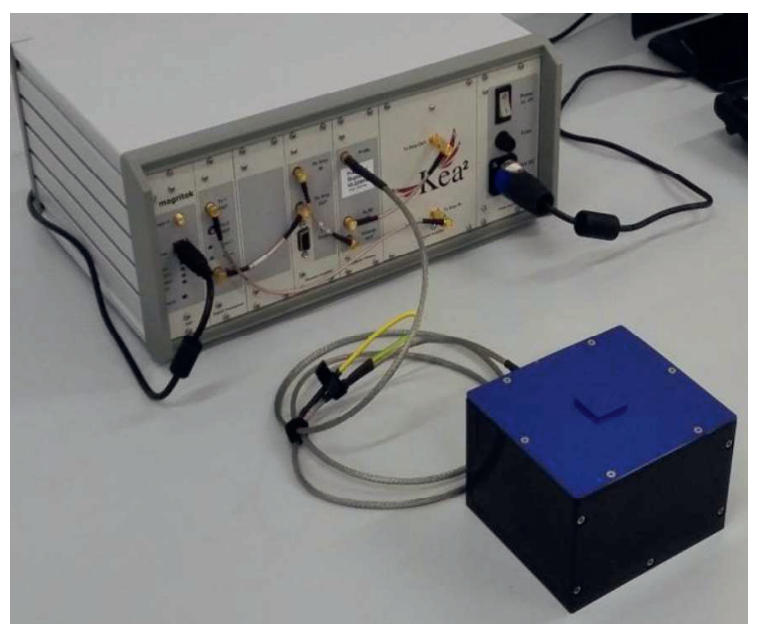

Fig. 1: Single-sided NMR system (Magritek NMRMouse PM5) and spectrometer KEA ${ }^{2}$.

To perform depth resolved measurements in different depth positions the samples were placed on polymethylmethacrylate (PMMA) plates with different thicknesses and a cutout for the rf coil. Moreover, cover glasses with 100, 150, 200 and $500 \mu \mathrm{m}$ thickness were used to achieve different measurement depths. The 
sample temperature was monitored using a hand-held IR-thermometer.

\section{Sample Materials}

Within this work samples of PE-Xa and PE-Xc with different degrees of cross-linking have been investigated. For the manufacturing of the PE-Xa samples, PE-HD (Borealis BorPEX HE1878E) with varying peroxide (AkzoNobel Trigonox 145-E85) addition were extruded into a mold and pressed to sheets. The PE-Xc samples (Sabic HDPE M40053SE) were first pressed to sheets and afterwards cross-linked via electron radiation by BGS Beta-GammaService $\mathrm{GmbH} \&$ Co. KG. Because of the different processing procedures the PE-Xa and $\mathrm{PE}-\mathrm{Xc}_{\mathrm{c}}$ samples differ in their crystallinity. During the processing of PE-Xa fewer crystalline areas can form because of the crosslinked amorphous areas. In contrast the PE-Xc samples show typical crystallinities since the cross-linking of the amorphous regions happens in solid state.

Additionally, the curing behavior of selected thermosets (epoxy resin and unsaturated polyester resin from LAMILUX Heinrich Strunz Holding $\mathrm{GmbH} \&$ Co. $\mathrm{KG}$ ) and an twocomponent (2C) adhesive (DELO-DUOPOX AD840 from DELO Industrie Klebstoffe $\mathrm{GmbH}$ \& Co. $\mathrm{KGaA}$ ) was investigated. The systems were mixed according to the recommendation given by the manufacturer and observed until they were fully cured.

\section{Reference Methods}

To allow a quantification of the degree of crosslinking, the standard wet chemical analysis was used for the PE-X samples. It was carried out according to DIN EN ISO 10147 [2]. PE-X flakes were weighted and cooked in Xylene for $8 \mathrm{~h}$. Afterwards they were dried for at least $3 \mathrm{~h}$ and the remaining material is weighted again. The degree of cross-linking can be derived from eq. (1):

$D_{X}=\frac{m_{\text {remaining }}}{m_{\text {initial }}}$

Differential Scanning Calorimetry (DSC) was the method of choice to measure the crystallinity of the PE-X samples and also the curing state of the adhesive and resin systems at room temperature and certain times. The DSC experiments were carried out using a NETZSCH DSC 204 F1 Phoenix with standard sample pans.

Finally, Dielectric Analysis (DEA) allows the monitoring of the curing process of the resin and adhesive samples. A NETZSCH DEA 288 Epsilon with one way IDEX sensors was used.
The ion viscosity was measured at different temperatures and frequencies.

\section{Degree of Cross-Linking of PE-X}

The wet chemical analysis showed that the degree of cross-linking of PE-Xa increases with a higher amount of peroxide or in the case of $\mathrm{PE}-\mathrm{Xc}$ a higher energy dose, respectively. Concerning the manufacturing process of the PE-Xa samples, a certain inhomogeneity was expected, which was confirmed in form of high relative errors.

The DSC measurements showed that the crystallinity of PE-X samples decreases with an increasing degree of cross-linking, which is shown in Fig. 2. It is also possible to differentiate between the used PE-Xa and $P E-X c$ samples in the first heating, since PE-Xc samples show a nearly constant crystallinity because of their manufacturing process. This effect vanishes in the second heating after the crystalline areas have been molten and cannot form again because of the cross-linked amorphous regions like in the PE-Xa case.

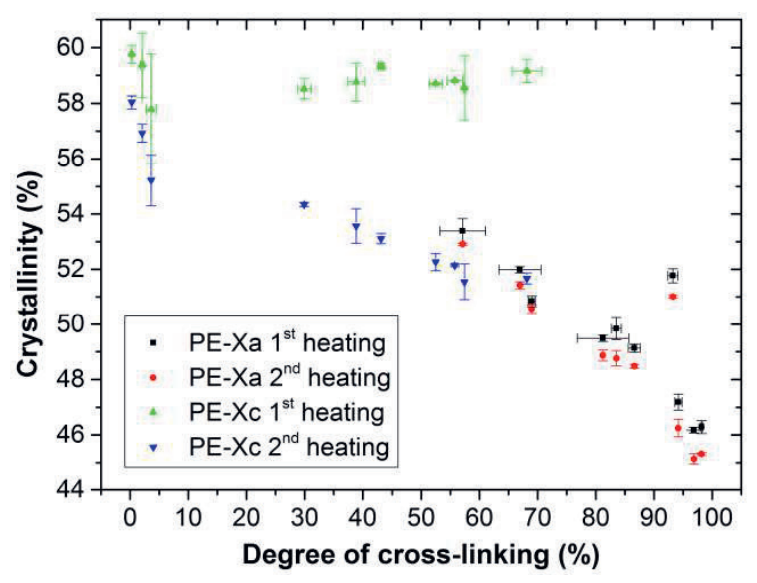

Fig. 2: Crystallinity of PE-Xa and PE-Xc samples measured with DSC. The degrees of cross-linking were derived from wet chemical analysis (DIN EN ISO 10147).

The measurements with the NMR-Mouse were conducted with CPMG pulse sequences at room temperature. The results were fitted with the bi-exponential function given by eq. (1). This non-restricted four-parameter fit allows many different solutions with the same result for relatively short $\mathrm{T}_{2}$ times of the PE-X samples. Therefore the more robust echo sum method, in the first step non-normalized, was used. The echo sums of PE-Xa and PE-Xc samples with different degrees of cross-linking are given in Fig. 3 and Fig. 4, respectively. It can be clearly seen, that the echo sums of the two PE-X types show different trends for increasing degrees of cross-linking. Generally, a decreasing echo sum would be expected since the cross-linking decreases the mobility of the polymer chains. 
For the PE-Xa samples the high amount of amorphous regions affects the measurement, since the measurable signals are mainly received from the amorphous regions. This effect seems to be stronger than the signal decrease based on the increased cross-linking. Correlating the degree of cross-linking with the echo sum the accuracy for a prediction may be possible in steps between $10-20 \%$, which is comparable to the results shown by Netto [9].

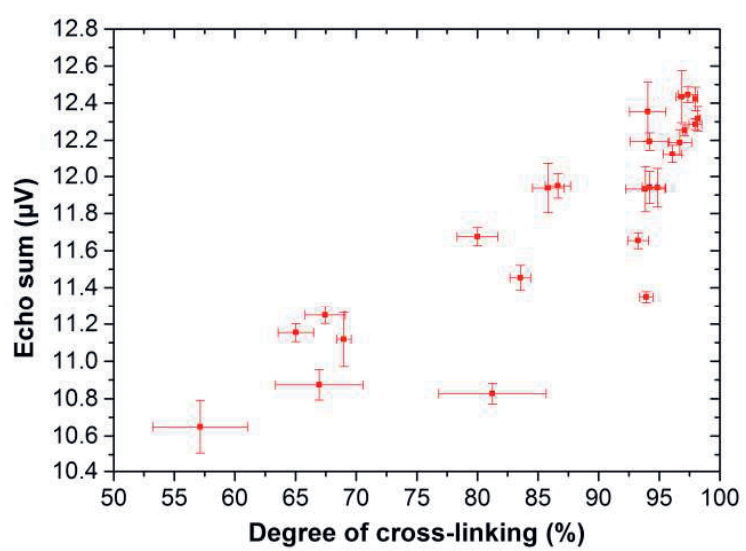

Fig. 3: Echo sum vs. degree of cross-linking of $P E-X a$ using CPMG pulse sequence.

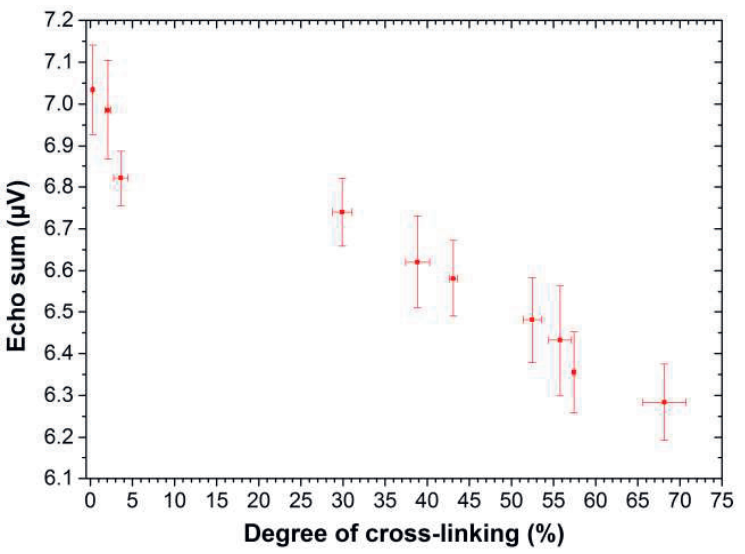

Fig. 4: Echo sum vs. degree of cross-linking of PE-XC using CPMG pulse sequence.

The scattering of the results for PE-Xa may be originated in the inhomogeneity of the samples. This effect was also determined when examining the general homogeneity of the samples at different lateral positions with the same measurement depth located in the center of the bulk. Here the PE-Xc samples show comparable values for the echo sum for different measurement positions. This is exemplarily shown in Fig. 5 for three different positions when viewing the square plate from the front. Moreover the equable homogeneity in depth direction was verified by measurements with distance steps of $200 \mu \mathrm{m}$ throughout the samples. The results of depth profiles of three different $\mathrm{PE}-\mathrm{XC}_{\mathrm{c}}$ samples measured at the center are depicted in Fig. 6.
Taking into account that only single measurements were conducted and assuming an uncertainty of glass spacer thickness $( \pm 20 \mu \mathrm{m})$, the deviation of the measured echo sums is of the same order (about $0.2 \mu \mathrm{V}$ ) as for the measurements in lateral direction. Measurements on the PE-Xa samples yield the same results, which is not shown here.

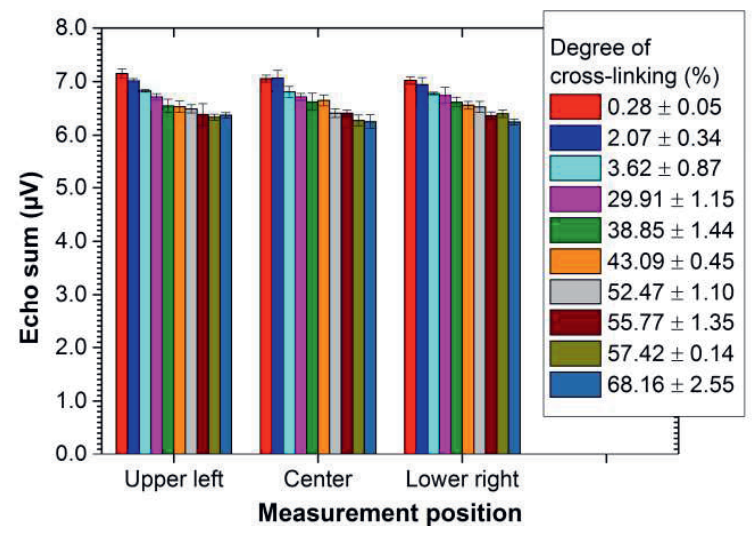

Fig. 5: Echo sums from different measurement positions of PE-Xc samples.

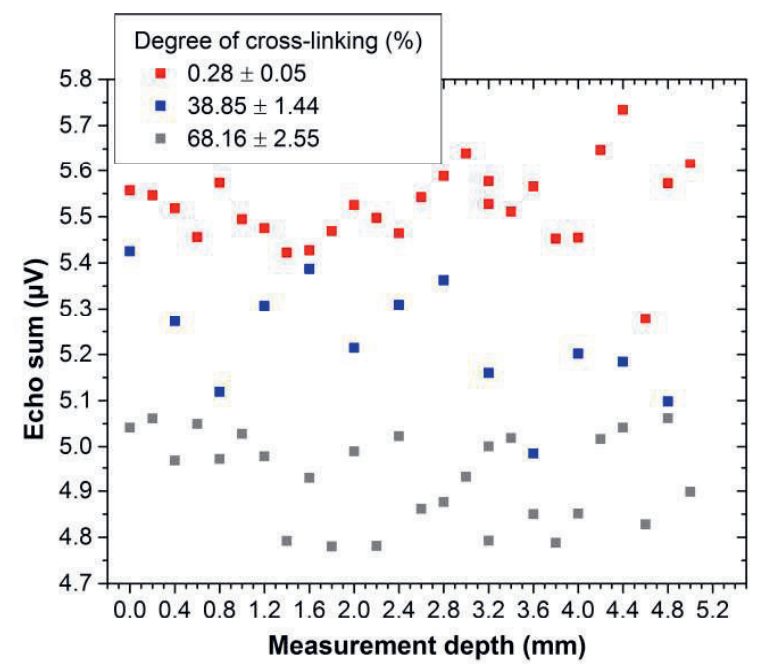

Fig. 6: Depth profiles of PE-Xc samples with different degrees of cross-linking measured in the center.

\section{Curing of Adhesives and Resins}

When monitoring the curing of thermoset samples with DSC, an exothermal peak can be measured during the first heating. The peak area is decreasing with increasing curing of the sample. In general this is a good indication for evaluating the degree of curing.

In DEA measurements the ion viscosity is observed at certain frequencies and temperatures and monitored during the curing process. In theory, the ion viscosity reaches a plateau when the sample is completely cured. However the time for reaching the plateau depends on the amount of material covering the IDEX sensor, the temperature as well as the used frequency. Therefore the curing times 
given by the material manufacturer are merely vague guide values.

For the NMR measurements the observed adhesive was mixed within a paper cup and put directly onto the NMR-Mouse, so that the sensitive volume was within the adhesive. Since the resin samples were heat activated in a heating furnace $\left(60-80^{\circ} \mathrm{C}\right)$, the use of paper cups was not practicable. Therefore the resin samples were placed in small sample cups made of polypropylene (PP) with no measurable signal contribution. They were also placed directly on the rf coil immediately after they were taken out of the furnace. The thermoset samples, especially in the uncured state, exhibited much higher signals than the PE-X samples. Therefore much better biexponential fits for the $T_{2}$ times were possible. Nonetheless, the graphs received when using echo sums are still smoother. The curing of the $2 \mathrm{C}$ epoxy adhesive at room temperature is illustrated in Fig. 7. The curing times provided by the manufacturer at room temperature $\left(23^{\circ} \mathrm{C}\right)$ are $7 \mathrm{~h}$ until firmness to touch and $16 \mathrm{~h}$ to receive functional strength. These times depend on the amount of material used. Since the volume in the paper cup was higher than for the reference curing times the different curing states were probably reached earlier.

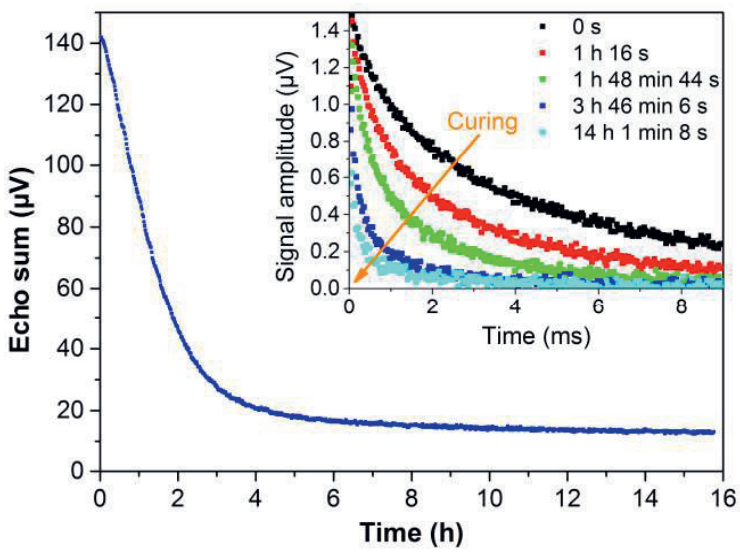

Fig. 7: Curing of a 2C epoxy adhesive (DELODUOPOX AD840) at room temperature. The inset shows single measurements at certain times.

The examined epoxy and unsaturated polyester resins showed similar trends with different curing times. For those materials an additional effect could be seen. At the beginning of the measurement, while the hot sample cups cooled down on the rf coil, an unexpected signal raise could be observed. This was probably caused by a temperature-based shift of $T_{1}$ times along with too short repetition times between measurements and hence lower maximum signals. Moreover also the position of the sensitive volume can shift, since it is dependent on the temperature of the magnets.
For a quantification of the degree of curing a user-defined $100 \%$ value is necessary. Currently, normalization allows the comparison of different measurements providing the same starting time. Also the slope of the decaying signal curve seems to be a good criterion for the reactivity of the thermosets.

\section{Discussion}

All materials examined here with the NMRMouse showed signal trends that can be used for a correlation with the degree of cross-linking and curing.

The different trends for PE-Xa and PE-Xc, probably based on different crystallinity levels, require further investigations. First step can be the tempering of the samples at temperatures above crystallite melting temperature. This should have the same influence as the second heating in the DSC measurements and result in lower crystallinity of the PE-Xc samples. Consequently, same signal trends in the NMR are expected after this treatment. Furthermore the loss in crystallinity caused by cross-linking during the manufacturing process (and maybe after tempering) can be correlated with the degree of cross-linking.

Moreover also the effects of sample and magnet temperature have to be taken into account. In general, a higher sample temperature will lead to a higher signal caused by increased molecular mobility but also the $T_{1}$ times can vary. On the other side, a constant magnet temperature is crucial, since shifts in the position of the sensitive volume and the resonance frequency can occur. For the experiments with resins and adhesives the amount of sample material was rather high. Testing with thin films could reduce the reaction temperature development and would, in the case of adhesives, be closer to the practical application. Besides active cooling with a fan or shock cooling of heated samples might be a possibility to freeze the actual curing state.

As shown for the PE-X samples, the measurement uncertainty is rather big and disallows a quantification of the degree of cross-linking in steps of (sub-)percent. The main reason for this is the inhomogeneous magnetic field. To achieve a higher signal-tonoise ratio and probably smaller measurement errors, longer measurement times and stable environmental conditions during the measurement are necessary. Also, for detailed observations, the use of systems with homogeneous magnetic field, like benchtop NMR, might deliver valuable additional information. However, the use of the singlesided system is still preferred, as non- 
destructive and non-contact testing is favorable for production monitoring.

\section{Summary and Outlook}

The experiments shown here with single-sided NMR on PE-X demonstrate the possibility to quantify the degree of cross-linking in PE-X. Some points like the different trends of $\mathrm{PE}-\mathrm{Xa}$ and $\mathrm{PE}-\mathrm{Xc}_{\mathrm{c}}$ and the possible resolution for quantification still have to be examined in detail. Adhesives and resins exhibited more signal than PE-X. Cure monitoring for these materials proved to be possible, when starting point and final cure are defined and comparable measurement conditions are provided. For further experiments, thin adhesive films (100 $200 \mu \mathrm{m}$ ) should be used to reduce reaction temperature caused by the amount of material and feature more industrial practice.

Another interesting topic is the examination of effects caused by higher sample and magnet temperature. Therefore the construction of a dummy system without magnets but the same geometry as the NMR-Mouse is planned to check for how long the system can be exposed to higher temperatures before the magnets reach the critical Curie temperature.

The relatively short measurement times of only a few seconds have the potential for realizing inline-monitoring systems for the degree of cross-linking during the production of semifinished products or the curing of thermosets (e. g. in composites) and adhesives on light weight construction.

\section{Acknowledgements}

The IGF project $18258 \mathrm{~N}$ by the research association "Fördergemeinschaft für das SKZ" was funded through the AiF within the scope of the program for the Promotion of Cooperative Industrial Research and Development (IGF) by the Federal Ministry for Economic Affairs and Energy due to a decree by the German Federal Parliament. We would like to thank for the financial support. Furthermore we would like to thank the members of the project-related committee for the discussions and DELO Industrie Klebstoffe $\mathrm{GmbH}$ \& $\mathrm{Co}$. $\mathrm{KGaA}$ and LAMILUX Heinrich Strunz Holding GmbH \& Co. KG for the supply of samples and BGS BetaGamma-Service GmbH \& Co. KG for the crosslinking of samples.

\section{References}

[1] T. A. Osswald, E. Baur, S. Brinkmann, K. Oberbach, E. Schmachtenberg, International Plastics Handbook, Carl Hanser publishing house, Munich (2006); doi: $10.3139 / 9783446407923$
[2] DIN Deutsches Institut für Normung e.V., DIN EN ISO 10147, Pipes and fittings made of crosslinked polyethylene (PE-X) - Estimation of the degree of crosslinking by determination of the gel content, Beuth publication house, Berlin (2013)

[3] B. Blümich, P. Blümler, G. Eidmann,

A. Guthausen, R. Haken, U. Schmitz, K. Saito, G. Zimmer, The NMR-mouse: construction, excitation, and applications, Magnetic Resonance Imaging 16, 479-484 (1998); doi: 10.1016/S0730-725X(98)00069-1

[4] F. Bloch, Nuclear induction, Physical Review 70 460-474 (1946); doi: 10.1103/PhysRev.70.460

[5] N. Bloembergen, E. M. Purcell, R. V. Pound, Relaxation effects in nuclear magnetic resonance absorption, Physical Review 73, 679-712 (1948); doi: 10.1103/PhysRev.73.679

[6] M. Dole, Cross-Linking and Crystallinity in Irradiated Polyethylene, Polymer-Plastics Technology and Engineering 13(1), 41-64 (1979); doi: $10.1080 / 03602557908067674$

[7] C. G. Fry, A. C. Lind, Determination of crosslink density in thermoset polymers by use of solidstate proton NMR techniques, Macromolecules 21(5), 1292-1297 (1988); doi: $10.1021 / \mathrm{ma00183a019}$

[8] H. Hirabayashi, A. Iguchi, K. Yamada, H. Nishimura, K. Ikawa, H. Honma, Study on the Structure of Peroxide Cross-Linked Polyethylene Pipes with Several Stabilizers, Materials Sciences and Applications 4, 497-503 (2013); doi: $10.4236 /$ msa.2013.49060

[9] A. M. Netto, Advances in Single-Sided NMR of Polymer and Cultural Heritage Science, PhD Thesis, Shaker publishing house, Aachen (2014)

[10] E. L. Hahn, Spin echoes, Physical Review 80 (1950) 580-594 (1950); doi: 10.1103/PhysRev.80.580

[11] H. Y. Carr, E. M. Purcell, Effects of diffusion on free precession in nuclear magnetic resonance experiments, Physical Review 94, 630-638 (1954); doi: 10.1103/PhysRev.94.630

[12] S. Meiboom, D. Gill, Modified spin-echo method for measuring nuclear relaxation times, Review of Scientific Instruments 29, 688-691 (1958); doi: $10.1063 / 1.1716296$

[13] B. Blümich, J. Pero, F. Casanova, Mobile singlesided NMR, Progress in Nuclear Magnetic Resonance Spectroscopy 52, 197-269 (2008); doi:10.1016/j.pnmrs.2007.10.002 